ITEP-TH-31/04

HMI-04-06

TCD-MATH-05-01

\title{
On a Class of Representations of Quantum Groups
}

\author{
A. Gerasimov \\ Institute for Theoretical \& Experimental Physics, 117259, Moscow, Russia \\ Department of Pure and Applied Mathematics, Trinity College, Dublin 2, Ireland \\ Hamilton Mathematics Institute, TCD, Dublin 2, Ireland \\ S. Kharchev ${ }^{1}$, \\ Institute for Theoretical \& Experimental Physics, 117259, Moscow, Russia \\ D. Lebedev ${ }^{2}$ \\ Institute for Theoretical \& Experimental Physics, 117259, Moscow, Russia and \\ Max-Planck-Institut fur Mathematik, Vivatsgasse 7, D-53111 Bonn, Germany, \\ S. Oblezin ${ }^{3}$ \\ Institute for Theoretical \& Experimental Physics, 117259, Moscow, Russia
}

\begin{abstract}
This paper is a short account of the construction of a new class of the infinitedimensional representations of the quantum groups. The examples include finitedimensional quantum groups $U_{q}(\mathfrak{g})$, Yangian $Y(\mathfrak{g})$ and affine quantum groups at zero level $U_{q}(\hat{\mathfrak{g}})_{c=0}$ corresponding to an arbitrary finite-dimensional semisimple Lie algebra $\mathfrak{g}$. At the intermediate step we construct the embedding of the quantum groups into the algebra of the rational functions on the quantum multi-dimensional torus. The explicit parameterization of the quantum groups used in this paper turns out to be closely related to the parameterization of the moduli spaces of the monopoles. As a result the proposed constructions of the representations provide a quantization of the moduli spaces of the monopoles on $\mathbb{R}^{3}$ and $\mathbb{R}^{2} \times S^{1}$.
\end{abstract}

\footnotetext{
${ }^{1}$ E-mail: kharchev@itep.ru

${ }^{2}$ E-mail: lebedev@mpim-bonn.mpg.de

${ }^{3}$ E-mail: Sergey.Oblezin@itep.ru
} 


\section{Introduction}

Constructions of explicit realizations of irreducible representations are not only interesting from the general point of view but also play a key role in the applications of the Representation theory to Number theory, Geometry and Physics. "Good" realizations often capture important features of the underlying object and thus lead to a better understanding of its properties.

Below we describe a construction of the infinite-dimensional representations of the quantum groups that may be considered as a generalization of the well-known construction of the finite-dimensional representations of the classical group due to Gelfand-Zetlin [12, [13]. The authors came to this construction while trying to understand some explicit integral representations of the wave function of the quantum integrable theories [20, [21] obtained in the framework of Quantum Inverse Scattering Method (QISM) [8], 22]. As it is in all other constructions of the irreducible representations, the crucial step is the choice of appropriate coordinates on Lie groups and maximal commutative subalgebras in (the skew-filed of fractions of) the universal enveloping algebra. Late, this construction was generalized to the case of the quantum groups $U_{q}(\mathfrak{g l}(N))$ [16]. The obtained realization of the representation of the finite-dimensional quantum group possess very interesting properties. In particular, the representation space has a natural structure of $U_{q}(\mathfrak{g}) \otimes U_{q^{\vee}}\left(\mathfrak{g}^{\vee}\right)$ - bimodule where $U_{q^{\vee}}\left(\mathfrak{g}^{\vee}\right)$ is dual algebra. This duality turns out to be closely related to Langlands duality.

It would be natural to suspect that further generalizations of the proposed constructions to the infinite-dimensional algebras are possible. Thus in [17] the construction of some class of the representations of the Yangian $Y(\mathfrak{g})$ for an arbitrary finite-dimensional semisimple Lie algebra $\mathfrak{g}$ was given. These representations arise as a quantization of the symplectic leaves of the classical counterpart of the Yangian. Surprisingly it turns out that the symplectic leaves of the classical Yangian coincide with the moduli spaces of the monopoles supplied with the appropriate symplectic structure. The corresponding explicit expressions for the symplectic structure on the moduli spaces of $G$-monopoles were derived in [1] for $G=S U(2)$, in [4] for $G=S U(N)$ and in [11] for the general case. Thus the proposed in [17 construction of the representations of Yangian provides at the same time the quantization of the moduli space of monopoles. This connection between the variables arising in the context of QISM and the variables arising in the study of the monopoles using the twistor methods [1] is quite remarkable and obviously is a particular manifestation of the deep relationship between these two subjects.

In this note we further generalize the construction of [14, [16, [17] to obtain a realization of a class of the infinite-dimensional representations of the finite-dimensional quantum groups $U_{q}(\mathfrak{g})$ and affine quantum groups $U_{q}(\hat{\mathfrak{g}})_{c=0}$ with zero level $c=0$ for an arbitrary semi-simple Lie algebra $\mathfrak{g}$. As an intermediate step, we construct the embedding of quantum groups into the algebra of rational functions on the quantum multi-dimensional torus. We postpone the technical details to another occasion [18] and concentrate on the explicit expressions for the representations of the quantum groups.

Similar to the connection of the Yangian representations with the quantization of the monopoles on $\mathbb{R}^{3}$ the proposed representations of the affine algebra are connected with the 
quantization of the periodic monopoles on $\mathbb{R}^{2} \times S^{1}$. In particular the classification of the trigonometric $r$-matrices underlying the quantum affine algebras $U_{q}(\hat{\mathfrak{g}})[\underline{3}$. corresponds to the classification of the special class of asymptotic boundary conditions on a monopole solutions on $\mathbb{R}^{2} \times S^{1}$. It would be natural to make one step further and consider the quantization of the moduli space of the double-periodic monopoles on $\mathbb{R} \times S^{1} \times S^{1}$. Presumably this should correspond to the quantum elliptic algebras and, indeed, the choice of the asymptotic boundary conditions may be associated with elliptic $r$-matrix [3]. The detailed account of the relevant description of the moduli spaces of monopoles on $\mathbb{R}^{2} \times S^{1}$ and $\mathbb{R} \times S^{1} \times S^{1}$ and its relation to the representations of the quantum groups will be given elsewhere [18].

Finally note that the embedding of $U_{q}(\mathfrak{g})$ into non-commutative multi-dimensional torus proposed in this note differs from the known similar constructions [24], 25], [2]. Also there is an obvious similarity of our constructions with the constructions in [9], [10. However results presented in this note seem to be new.

The plan of the paper is as follows. In Section 2 we recall the construction of a certain class of representations of the Yangian introduced in [17]. In Section 3 we give the generalization of the construction discussed in Section 2 to the case of the universal enveloping of the quantum affine algebra $U_{q}(\hat{\mathfrak{g}})$ at $c=0$ for an arbitrary semisimple Lie algebra $\mathfrak{g}$. In Section 4 the explicit construction of the representations of the quantum groups $U_{q}(\mathfrak{g})$ for an arbitrary semisimple Lie algebra $\mathfrak{g}$ is given.

Acknowledgments: The authors are grateful to M. Finkelberg, V. Gorbunov, A. Levin and A. Rosly for useful discussions. The research was partly supported by grants CRDF RM1-2545; INTAS 03-513350; grant NSh 1999.2003.2 for support of scientific schools, and by grants RFBR-03-02-17554 (A. Gerasimov, D. Lebedev, S. Oblezin), and RFBR-03-0217373 (S. Kharchev). The research of A. Gerasimov was also partly supported by SFI Basic Research Grant. D. Lebedev and S. Oblezin would like to thank the Max-Planck-Institut für Mathematik for support and warm hospitality. The research of S. Oblezin was also partly supported as a Independent University Möbius Prize Fellow; also S. Oblezin is thankful to the Jumelage (Twinship) Program CNRS-IUM for partial support and to l'Université d'Angers for the hospitality.

\section{A representation of $Y(\mathfrak{g})$}

In this section we remind the explicit construction of a class of representations of the Yangian in terms of difference operators given in [17.

We start with the definition of the Yangian for a semisimple Lie algebra $\mathfrak{g}$ due to Drinfeld 7]. Let $\mathfrak{h} \subset \mathfrak{b} \subset \mathfrak{g}$ be a simple finite-dimensional Lie algebra $\mathfrak{g}$ of rank $\ell$ over $\mathbb{C}$ with a fixed Cartan subalgebra $\mathfrak{h}$ and a Borel subalgebra $\mathfrak{b}$. Let $a=\left\|a_{i j}\right\|, i, j=1, \ldots, \ell$ be the Cartan matrix of $\mathfrak{g}, \Gamma$ be the set of vertices of the Dynkin diagram of $\mathfrak{g},\left\{\alpha_{i} \in \mathfrak{h}^{*}, i \in \Gamma\right\}$ be the set of simple roots and $\left\{\alpha_{i}^{\vee}, i \in \Gamma\right\}$ be the set of the corresponding coroots such that $a_{i j}=\alpha_{i}^{\vee}\left(\alpha_{j}\right)$. There exist coprime positive integers $d_{1}, \ldots, d_{\ell}$ such that the matrix $\left\|d_{i} a_{i j}\right\|$ is symmetric. Define the invariant bilinear form on $\mathfrak{h}^{*}$ by $\left(\alpha_{i}, \alpha_{j}\right)=d_{i} a_{i j}$, then $a_{i j}=\frac{2\left(\alpha_{i}, \alpha_{j}\right)}{\left(\alpha_{i}, \alpha_{i}\right)}$. 
Introduce the formal generating series $H_{i}(u), E_{i}(u), F_{i}(u), i \in \Gamma$ :

$$
\begin{gathered}
H_{i}(u)=1+\sum_{n=0}^{\infty} H_{i}^{(n)} u^{-n-1} \\
E_{i}(u)=\sum_{n=0}^{\infty} E_{i}^{(n)} u^{-n-1}, \quad F_{i}(u)=\sum_{n=0}^{\infty} F_{i}^{(n)} u^{-n-1} .
\end{gathered}
$$

Definition 2.1 The Yangian $Y(\mathfrak{g})$ is the associative algebra with the elements $H_{i}^{(n)}, E_{i}^{(n)}$, $F_{i}^{(n)}, i \in \Gamma ; n=0,1,2 \ldots$ and the following defining relations

$$
\begin{aligned}
& {\left[H_{i}(u), H_{j}(v)\right]=0 \text {, }} \\
& {\left[H_{i}(u), E_{j}(v)\right]=-\frac{\imath \hbar}{2}\left(\alpha_{i}, \alpha_{j}\right) \frac{\left[H_{i}(u), E_{j}(u)-E_{j}(v)\right]_{+}}{u-v},} \\
& {\left[H_{i}(u), F_{j}(u)\right]=\frac{\imath \hbar}{2}\left(\alpha_{i}, \alpha_{j}\right) \frac{\left[H_{i}(u), F_{j}(u)-F_{j}(v)\right]_{+}}{u-v},} \\
& {\left[E_{i}(u), F_{j}(v)\right]=-\imath \hbar \frac{H_{i}(u)-H_{i}(v)}{u-v} \delta_{i, j},} \\
& {\left[E_{i}(u), E_{i}(v)\right]=-\frac{\imath \hbar}{2}\left(\alpha_{i}, \alpha_{i}\right) \frac{\left(E_{i}(u)-E_{i}(v)\right)^{2}}{u-v}} \\
& {\left[F_{i}(u), F_{i}(v)\right]=\frac{\imath \hbar}{2}\left(\alpha_{i}, \alpha_{i}\right) \frac{\left(F_{i}(u)-F_{i}(v)\right)^{2}}{u-v},} \\
& {\left[E_{i}(u), E_{j}(v)\right]=} \\
& -\frac{\imath \hbar}{2}\left(\alpha_{i}, \alpha_{j}\right) \frac{\left[E_{i}(u), E_{j}(u)-E_{j}(v)\right]_{+}}{u-v}-\frac{\left[E_{i}^{(0)}, E_{j}(u)-E_{j}(v)\right]}{u-v} \\
& {\left[F_{i}(u), F_{j}(v)\right]=} \\
& \frac{\imath \hbar}{2}\left(\alpha_{i}, \alpha_{j}\right) \frac{\left[F_{i}(u), F_{j}(u)-F_{j}(v)\right]_{+}}{u-v}-\frac{\left[F_{i}^{(0)}, F_{j}(u)-F_{j}(v)\right]}{u-v}, \\
& \sum_{\sigma \in \mathfrak{S}_{m}}\left[E_{i}\left(u_{\sigma(1)}\right),\left[E_{i}\left(u_{\sigma(2)}\right), \ldots,\left[E_{i}\left(u_{\sigma(m)}\right), E_{j}(v)\right] \ldots\right]\right]=0 \\
& \sum_{\sigma \in \mathfrak{S}_{m}}\left[F_{i}\left(u_{\sigma(1)}\right),\left[F_{i}\left(u_{\sigma(2)}\right), \ldots,\left[F_{i}\left(u_{\sigma(m)}\right), F_{j}(u)\right] \ldots\right]\right]=0 \\
& m=1-a_{i j} \quad \text { for } i \neq j,
\end{aligned}
$$

where $[a, b]_{+}:=a b+b a$ and summation in (2.3) is performed over permutation group $\mathfrak{S}_{m}$.

Let $Y(\mathfrak{b}) \subset Y(\mathfrak{g})$ be the subalgebra generated by $H_{i}(u), E_{i}(u), i \in \Gamma$. 
The explicit description of the representation of the Yangian in terms of difference operators is based on the choice of a large enough commutative subalgebra. We shall use the coefficients of the series $H_{i}(u)$ as the generators of this subalgebra. In the constructed representation $H_{i}^{(n)}$ will act by multiplication on the functions of some auxiliary variables. Thus to obtain representation of the Yangian we should find the representation of the other generators in terms of some difference operators acting on the same space of functions.

Let us introduce a set of variables $\left\{\gamma_{i, k} ; i \in \Gamma ; k=1, \ldots, m_{i}\right\}$, where $m_{i} \in \mathbb{N}$ and let $\mathcal{M}$ be the space of meromorphic functions in these variables. Define the following difference operators acting on $\mathcal{M}: \beta_{i, k}=e^{\imath \hbar d_{i} \frac{\partial}{\partial \gamma_{i, k}}}$. Below we use the convention $\prod_{s=j}^{k} f_{s}:=1$, for any $f_{s}$ if $k<j$.

Consider the operators

$$
\begin{aligned}
& H_{i}(u)=R_{i}(u) \frac{\prod_{j \neq i} \prod_{r=1}^{-a_{j i}} \prod_{p=1}^{m_{j}}\left(u-\gamma_{j, p}-\frac{\imath \hbar}{2}\left(\alpha_{i}+r \alpha_{j}, \alpha_{j}\right)\right)}{\prod_{p=1}^{m_{i}}\left(u-\gamma_{i, p}\right)\left(u-\gamma_{i, p}-\frac{\imath \hbar}{2}\left(\alpha_{i}, \alpha_{i}\right)\right)}, \\
& E_{i}(u)=d_{i}^{-1 / 2} \sum_{k=1}^{m_{i}} \frac{\prod_{j=i+1}^{\ell} \prod_{r=1}^{-a_{j i}} \prod_{p=1}^{m_{j}}\left(\gamma_{i, k}-\gamma_{j, p}-\frac{\imath \hbar}{2}\left(\alpha_{i}+r \alpha_{j}, \alpha_{j}\right)\right)}{\left(u-\gamma_{i, k}\right) \prod_{p \neq k}\left(\gamma_{i, k}-\gamma_{i, p}\right)} \beta_{i, k}^{-1}, \\
& F_{i}(u)=-d_{i}^{-1 / 2} \sum_{k=1}^{m_{i}} R_{i}\left(\gamma_{i, k}+\frac{\imath \hbar}{2}\left(\alpha_{i}, \alpha_{i}\right)\right) \times \\
& \frac{\prod_{j=1}^{i-1} \prod_{r=1}^{-a_{j i}} \prod_{p=1}^{m_{j}}\left(\gamma_{i, k}-\gamma_{j, p}-\frac{\imath \hbar}{2}\left(\alpha_{i}+r \alpha_{j}, \alpha_{j}\right)+\frac{\imath \hbar}{2}\left(\alpha_{i}, \alpha_{i}\right)\right)}{\left(u-\gamma_{i, k}-\frac{i \hbar}{2}\left(\alpha_{i}, \alpha_{i}\right)\right) \prod_{p \neq k}\left(\gamma_{i, k}-\gamma_{i, p}\right)} \beta_{i, k},
\end{aligned}
$$

where the rational functions $R_{i}(u)$ will be specified below.

Theorem 2.1 [17] (i). For any set of positive integers $\left\{m_{i}, i \in \Gamma\right\}$ satisfying the conditions $l_{i}:=\sum_{j=1}^{\ell} m_{j} a_{j i} \in \mathbb{Z}_{\geq 0}$, introduce the polynomials $R_{i}(u)=\prod_{s=1}^{l_{i}}\left(u-\nu_{i, s}\right)$, where $\left\{\nu_{i, s}, i \in \Gamma\right.$, $\left.s=1, \ldots, l_{i}\right\}$ is a set of arbitrary complex parameters. Then the operators (2.4)-(2.6) considered as formal power series in $u^{-1}$, define a representation of $Y(\mathfrak{g})$ in the space $\mathcal{M}$.

(ii). Let $\left\{m_{i}, i \in \Gamma\right\}$ be an arbitrary set of positive integers and $R_{i}(u)$ be rational functions of the form $R_{i}(u)=\prod_{s=1}^{l_{i}^{+}}\left(u-\nu_{i, s}^{+}\right) / \prod_{s=1}^{l_{i}^{-}}\left(u-\nu_{i, s}^{-}\right)$, where $\left\{\nu_{i, s}^{ \pm}, i \in \Gamma, s=1, \ldots, l_{i}^{ \pm}\right\}$is a set of arbitrary complex parameters and $l_{i}^{+}-l_{i}^{-}=\sum_{j=1}^{\ell} m_{j} a_{j i}$. Then the operators (2.4), (2.5) considered as formal power series in $u^{-1}$, define a representation of $Y(\mathfrak{b})$ in the space $\mathcal{M}$.

Below we generalize this construction to the case of the quantum affine algebra at zero level. 


\section{A representation of $U_{q}(\hat{\mathfrak{g}})_{c=0}$}

In this section we construct a representation of the quantum affine algebra at the zero level $U_{q}(\hat{\mathfrak{g}})_{c=0}$. The construction is the direct generalization of the construction for the Yangian described in the previous section. We start with the definition of the quantum affine algebra $U_{q}(\hat{\mathfrak{g}})$ at $c=0$ for any semisimple Lie algebra $\mathfrak{g}$ in terms of generating series following [7].

Let $q$ be an undeterminate. Quantum affine algebra as an associative $\mathbb{C}(q)$-algebra may be present in terms of the elements $K_{i}^{ \pm 1}, H_{i}^{(n)}, n \in \mathbb{Z} \backslash\{0\}, E_{i}^{(n)}, F_{i}^{(n)}, n \in \mathbb{Z}, i \in \Gamma$ and relations. Introduce the formal generating series $K_{i}^{ \pm}(z), E_{i}(z)$, and $F_{i}(z), i \in \Gamma$ :

$$
\begin{aligned}
& K_{i}^{ \pm}(z)=K_{i}^{ \pm 1} \exp \left( \pm\left(q_{i}-q_{i}^{-1}\right) \sum_{n \in \mathbb{N}} H_{i}^{( \pm n)} z^{\mp n}\right) \\
& E_{i}(z)=\sum_{n \in \mathbb{Z}} E_{i}^{(n)} z^{-n}, \quad F_{i}(z)=\sum_{n \in \mathbb{Z}} F_{i}^{(n)} z^{-n}
\end{aligned}
$$

where $q_{i}:=q^{d_{i}}$.

Definition 3.1 Quantum affine algebra $U_{q}(\hat{\mathfrak{g}})$ at $c=0$ is the associative algebra with elements $K_{i}^{ \pm 1}, H_{i}^{(n)}, n \in \mathbb{Z} \backslash\{0\} ; E_{i}^{(n)}, F_{i}^{(n)}, n \in \mathbb{Z} ; i \in \Gamma$ and the following defining relations:

$$
\begin{aligned}
& K_{i}^{ \pm}(z) K_{j}^{ \pm}(w)=K_{j}^{ \pm}(w) K_{i}^{ \pm}(z), \\
& K_{i}^{+}(z) K_{j}^{-}(w)=K_{j}^{-}(w) K_{i}^{+}(z), \\
& \left(z-q_{i}^{a_{i j}} w\right) K_{i}^{ \pm}(z) E_{j}(w)=\left(q_{i}^{a_{i j}} z-w\right) E_{j}(w) K_{i}^{ \pm}(z), \\
& \left(z-q_{i}^{-a_{i j}} w\right) K_{i}^{ \pm}(z) F_{j}(w)=\left(q_{i}^{-a_{i j}} z-w\right) F_{j}(w) K_{i}^{ \pm}(z), \\
& {\left[E_{i}(z), F_{j}(w)\right]=\frac{\delta_{i, j}}{q_{i}-q_{i}^{-1}} \delta(z / w)\left(K_{i}^{+}(w)-K_{i}^{-}(z)\right),} \\
& \left(z-q_{i}^{a_{i j}} w\right) E_{i}(z) E_{j}(w)=\left(q_{i}^{a_{i j}} z-w\right) E_{j}(w) E_{i}(z), \\
& \left(z-q_{i}^{-a_{i j}} w\right) F_{i}(z) F_{j}(w)=\left(q_{i}^{-a_{i j}} z-w\right) F_{j}(w) F_{i}(z), \\
& \sum_{\sigma \in \mathfrak{S}_{m}} \sum_{k=0}^{m}(-1)^{k}\left[\begin{array}{c}
m \\
k
\end{array}\right]_{q_{i}} E_{i}\left(z_{\sigma(1)}\right) \ldots E_{i}\left(z_{\sigma(k)}\right) E_{j}(w) E_{i}\left(z_{\sigma(k+1)}\right) \ldots E_{i}\left(z_{\sigma(m)}\right)=0, \\
& \sum_{\sigma \in \mathfrak{S} m} \sum_{k=0}^{m}(-1)^{k}\left[\begin{array}{c}
m \\
k
\end{array}\right]_{q_{i}} F_{i}\left(z_{\sigma(1)}\right) \ldots F_{i}\left(z_{\sigma(k)}\right) E_{j}(w) F_{i}\left(z_{\sigma(k+1)}\right) \ldots F_{i}\left(z_{\sigma(m)}\right)=0 \\
& m=1-a_{i j} \quad \text { for } i \neq j .
\end{aligned}
$$

Here we use the standard notations $\left[\begin{array}{c}m \\ k\end{array}\right]_{q}=\frac{[m]_{q} !}{[k]_{q} ![m-k]_{q} !},[k]_{q} !=\prod_{1 \leq j \leq k} \frac{q^{j}-q^{-j}}{q-q^{-1}}$. The formal delta-function is defined as $\delta(z)=\sum_{n \in \mathbb{Z}} z^{n}$. For more details on the operator-valued formal series see, for example, [19]. 
To define a representation of $U_{q}(\hat{\mathfrak{g}})_{c=9}$ in terms of the difference operators, we start with construction of the embedding of the corresponding universal enveloping algebra into the algebra $\mathbb{T}_{q}$ of the rational functions of the non-commutative multi-dimensional torus.

Let $\mathbb{T}_{q}$ be the associative $\mathbb{C}(q)$-algebra of the rational functions of invertible elements $\mathbf{v}_{i, k}, \mathbf{u}_{i, k}, \mathbf{w}_{i, s}, i=1, \ldots, \ell ; k=1, \ldots, m_{i} \in \mathbb{N} ; s=1, \ldots, l_{i} \in \mathbb{Z}_{\geq 0}$, subject to relations

$$
\begin{gathered}
\mathbf{v}_{i, k} \mathbf{v}_{j, l}=\mathbf{v}_{j, l} \mathbf{v}_{i, k}, \quad \mathbf{u}_{i, k} \mathbf{u}_{j, l}=\mathbf{u}_{j, l} \mathbf{u}_{i, k}, \\
\mathbf{u}_{i, k} \mathbf{v}_{j, l}=q_{i}^{\delta_{i, j} \delta_{k, l}} \mathbf{v}_{j, l} \mathbf{u}_{i, k},
\end{gathered}
$$

and $\mathbf{w}_{i, s}$ are central elements in $\mathbb{T}_{q}$. Chose the set of natural numbers $\left\{m_{i}\right\}$ satisfying the conditions

$$
\sum_{j=1}^{\ell} m_{j} a_{j i}=l_{i}
$$

and consider the following formal generating functions $K_{i}^{ \pm}, E_{i}(z)$ and $F_{i}(z)$ in variable $z$.

The functions $K_{i}^{+}(z)$ and $K_{i}^{-}(z)$ are defined as infinite series expansion in $z^{-1}$ and $z$ of the same rational function

$$
K_{i}(z)=c_{i} \prod_{j=1}^{\ell} \prod_{p=1}^{m_{j}} \mathbf{v}_{j, p}^{a_{j i}} \cdot \prod_{s=1}^{l_{i}}\left(z \mathbf{w}_{i, s}^{-1}-\mathbf{w}_{i, s}\right) \frac{\prod_{j \neq i} \prod_{r=1}^{-a_{j i}} \prod_{p=1}^{m_{j}}\left(z-q_{j}^{a_{j i}+2 r} \mathbf{v}_{j, p}^{2}\right)}{\prod_{p=1}^{m_{i}}\left(z-\mathbf{v}_{i, p}^{2}\right)\left(z-q_{i}^{2} \mathbf{v}_{i, p}^{2}\right)},
$$

where $c_{i}=\prod_{j=1}^{\ell} q_{j}^{m_{j} a_{j i} / 2}$. Due to conditions (3.4) the degrees of the polynomials in the numerator and in the denominator in (3.5) coincide and thus $K_{i}^{ \pm}(z)$ as the power series in $z^{\mp 1}$ have the form (3.1) where $K_{i}=c_{i} \prod_{s=1}^{l_{i}} \mathbf{w}_{i, s}^{-1} \cdot \prod_{j=1}^{\ell} \prod_{p=1}^{m_{j}} \mathbf{v}_{j, p}^{a_{j i}}$.

Let $R^{( \pm)}(z)$ be the polynomials such that $R_{i}^{(+)}(z) R_{i}^{(-)}(z)=\prod_{s=1}^{l_{i}}\left(z \mathbf{w}_{i, s}^{-1}-\mathbf{w}_{i, s}\right)$. The other generating series are defined as follows

$$
\begin{gathered}
E_{i}(z)=\frac{c_{i}}{q_{i}-q_{i}^{-1}} \prod_{p=1}^{m_{i}} \mathbf{v}_{i, p} \cdot \prod_{j=i+1}^{\ell} \prod_{p=1}^{m_{j}} \mathbf{v}_{j, p}^{a_{j i}} \cdot \\
\sum_{k=1}^{m_{i}} \delta\left(z / \mathbf{v}_{i, k}^{2}\right) \mathbf{v}_{i, k}^{-2} R_{i}^{(+)}\left(\mathbf{v}_{i k}^{2}\right) \frac{\prod_{j=i+1}^{\ell} \prod_{r=1}^{-a_{j i}} \prod_{p=1}^{m_{j}}\left(\mathbf{v}_{i, k}^{2}-q_{j}^{a_{j i}+2 r} \mathbf{v}_{j, p}^{2}\right)}{\prod_{p \neq k}\left(\mathbf{v}_{i, k}^{2}-\mathbf{v}_{i, p}^{2}\right)} \mathbf{u}_{i, k}^{-1}, \\
F_{i}(z)=-\frac{q_{i}^{-2 m_{i}}}{q_{i}-q_{i}^{-1}} \prod_{p=1}^{m_{i}} \mathbf{v}_{i, p} \cdot \prod_{j=1}^{i-1} \prod_{p=1}^{m_{j}} \mathbf{v}_{j, p}^{a_{j i}} \cdot \\
\sum_{k=1}^{m_{i}} \delta\left(z / q_{i}^{2} \mathbf{v}_{i, k}^{2}\right) \mathbf{v}_{i, k}^{-2} R_{i}^{(-)}\left(q_{i}^{2} \mathbf{v}_{i, k}^{2}\right) \frac{\prod_{j=1}^{i-1} \prod_{r=1}^{-a_{j i}} \prod_{p=1}^{m_{j}}\left(q_{i}^{2} \mathbf{v}_{i, k}^{2}-q_{j}^{a_{j i}+2 r} \mathbf{v}_{j, p}^{2}\right)}{\prod_{p \neq k}\left(\mathbf{v}_{i, k}^{2}-\mathbf{v}_{i, p}^{2}\right)} \mathbf{u}_{i, k} .
\end{gathered}
$$


Theorem 3.1 For any set of positive integers $\left\{m_{i}, i \in \Gamma\right\}$ obeying the conditions (3.4), the generating series $K_{i}^{ \pm}(z), E_{i}(z)$, and $F_{i}(z)$ defined by (3.5), (3.6), satisfy the relations (3.2) and therefore define an embedding $\pi: U_{q}(\hat{\mathfrak{g}})_{c=0} \hookrightarrow \mathbb{T}_{q}$.

The proof will be given in [18].

Let us introduce a set of variables $\left\{\gamma_{i, k} \in \mathbb{C} ; i \in \Gamma ; k=1, \ldots, m_{i}\right\}$, and let $\mathcal{M}$ be the space of meromorphic functions in these variables. We also fix a set of the complex numbers $\nu_{i, s}, i=1, \ldots, \ell, s=1, \ldots, l_{i}$. Define the following representation of $\mathbb{T}_{q}$ in terms of difference operators acting on $\mathcal{M}$ :

$$
\mathbf{u}_{i, k}=e^{\imath \omega_{1} d_{i} \frac{\partial}{\partial \gamma_{i, k}}}, \quad \mathbf{v}_{i, k}=e^{\frac{2 \pi \gamma_{i k}}{\omega_{2}}}, \quad \mathbf{w}_{i, s}=e^{\frac{2 \pi \nu_{i, s}}{\omega_{2}}}, \quad q=e^{\frac{2 \pi i \omega_{1}}{\omega_{2}}},
$$

where $\omega_{1}$ and $\omega_{2}$ are arbitrary complex parameters. The following proposition is the simple consequence of Theorem 3.1 .

Proposition 3.1 Let us given the representation of $\mathbb{T}_{q}$ defined by 3.7). The coefficients $K_{i}^{ \pm 1}, H_{i}^{(n)}, n \in \mathbb{Z} \backslash\{0\}, E_{i}^{(n)}, F_{i}^{(n)}, n \in \mathbb{Z}, i \in \Gamma$ of the formal power series (3.5), (3.6) define a representation of $U_{q}(\hat{\mathfrak{g}})_{c=0}$ in $\mathcal{M}$.

\section{A representation of $U_{q}(\mathfrak{g})$}

The finite-dimensional quantum groups $U_{q}(\mathfrak{g})$ may be naturally considered as the subalgebras of the affine quantum groups $U_{q}(\hat{\mathfrak{g}})_{c=0}$. Indeed, the generators $K_{i}^{ \pm 1}, E_{i}^{(0)}$, and $F_{i}^{(0)}$ introduced in the previous section obey the commutation relations of the finite-dimensional quantum group $U_{q}(\mathfrak{g})$. We shall omit the superscript $(0)$ and write down simply $E_{i}$ and $F_{i}$. Thus the representations of the affine quantum groups introduced above automatically provide the representations of the finite-dimensional quantum groups. Below we give the explicit expressions for the generators in this representations. However we would prefer to work in this section in a slightly more general case and consider the various rational forms of the quantum groups. Let $Q$ and $P$ be the root and weight lattices of the Lie algebra $\mathfrak{g}$. There are different rational forms of the quantum group not isomorphic as $\mathbb{C}(q)$ algebras which may be enumerated by the choice of a sublattice $M$ such that $Q \subseteq M \subseteq P$ (see [5], 23] and references therein). Thus for example the adjoint (the smallest) rational form $U_{q}^{Q}(\mathfrak{g})$ is the associative $\mathbb{C}(q)$-algebra generated by $E_{i}, F_{i}$ and $K_{i}^{ \pm}, i \in \Gamma$ with the defining relations:

$$
\begin{gathered}
K_{i} K_{i}^{-1}=K_{i}^{-1} K_{i}=1, \quad K_{i} K_{j}=K_{j} K_{i}, \\
K_{i} E_{j} K_{i}^{-1}=q_{i}^{a_{i j}} E_{j}, \quad K_{i} F_{j} K_{i}^{-1}=q_{i}^{-a_{i j}} F_{j}, \\
E_{i} F_{j}-F_{j} E_{i}=\delta_{i, j} \frac{K_{i}-K_{i}^{-1}}{q_{i}-q_{i}^{-1}}, \\
\sum_{r=0}^{1-a_{i j}}(-1)^{r}\left[\begin{array}{c}
1-a_{i j} \\
r
\end{array}\right]_{q_{i}} E_{i}^{1-a_{i j}-r} E_{j} E_{i}^{r}=0, \quad i \neq j, \\
\sum_{r=0}^{1-a_{i j}}(-1)^{r}\left[\begin{array}{c}
1-a_{i j} \\
r
\end{array}\right]_{q_{i}} F_{i}^{1-a_{i j}-r} F_{j} F_{i}^{r}=0, \quad i \neq j .
\end{gathered}
$$


On the other hand, the largest, simply-connected, rational from $U_{q}^{P}(\mathfrak{g})$ is obtained by adjoining to $U_{q}^{Q}(\mathfrak{g})$ the invertible elements $L_{i}, i \in \Gamma$, such that $K_{i}=\prod_{j} L_{j}^{a_{j i}}$ and obeying the relations $L_{i} E_{j} L_{i}^{-1}=q_{i}^{\delta_{i, j}} E_{j}, L_{i} F_{j} L_{i}^{-1}=q_{i}^{-\delta_{i, j}} F_{j}$. Given any intermediate lattice $Q \subseteq M \subseteq P$, the corresponding rational form $U_{q}^{M}(\mathfrak{g})$ is obtained by adjoining to $U_{q}^{Q}(\mathfrak{g})$ the elements $K_{\beta_{i}}=\prod_{j} L_{j}^{n_{j i}}$ for any basis $\beta_{i}=\sum_{j} n_{j i} \lambda_{j} \in M, i \in \Gamma$, where $\lambda_{j}$ are the fundamental weights. For any simple root $\alpha_{i}$ let $\alpha_{i}=\sum_{j} m_{j i} \beta_{j}$. Then the generators $K_{i}$ are expressed by $K_{\beta_{j}}$ as $K_{i}=\prod_{j} K_{\beta_{j}}^{m_{j i}}$. Let $\left\|M_{i j}\right\|$ be the matrix inverse to $\left\|m_{i j}\right\|$ and $d=\operatorname{det}\left\|a_{i j}\right\|$. Let $\mathbb{T}_{q}$ be the quantum torus defined in the previous section. The following Theorem is a generalization of the embedding of the finite-dimensional quantum group into quantum torus obtained by the restriction of embedding of the affine quantum group described in Section 3.

Theorem 4.1 (i). Let $\mathfrak{g}$ be an arbitrary semisimple Lie algebra. Let $K_{\beta_{i}}^{ \pm 1}, E_{i}, F_{i}, i \in \Gamma$ be the generators of the rational form of quantum group $U_{q}^{M}(\mathfrak{g})$, associated with the lattice $M$ such that $Q \subseteq M \subseteq P$. The following expressions define the embedding $\pi: U_{q}^{M}(\mathfrak{g}) \hookrightarrow \mathbb{T}_{q}$ :

$$
\begin{gathered}
\pi\left(K_{\beta_{i}}\right)=c_{\beta_{i}} \prod_{j=1}^{\ell}\left(\prod_{s=1}^{l_{j}} \mathbf{w}_{j, s}^{-d M_{j i}} \cdot \prod_{p=1}^{m_{j}} \mathbf{v}_{j, p}^{n_{j i}}\right) \\
\pi\left(E_{i}\right)=\frac{c_{\alpha_{i}}}{q_{i}-q_{i}^{-1}} \prod_{p=1}^{m_{i}} \mathbf{v}_{i, p} \cdot \prod_{j=i+1}^{\ell} \prod_{p=1}^{m_{j}} \mathbf{v}_{j, p}^{a_{j i}} \cdot \\
\sum_{k=1}^{m_{i}} \mathbf{v}_{i, k}^{-2} R_{i}^{(+)}\left(\mathbf{v}_{i k}^{2}\right) \frac{\prod_{j=i+1}^{\ell} \prod_{r=1}^{-a_{j i}} \prod_{p=1}^{m_{j}}\left(\mathbf{v}_{i, k}^{2}-q_{j}^{a_{j i}+2 r} \mathbf{v}_{j, p}^{2}\right)}{\prod_{p \neq k}\left(\mathbf{v}_{i, k}^{2}-\mathbf{v}_{i, p}^{2}\right)} \mathbf{u}_{i, k}^{-1} \\
\pi\left(F_{i}\right)=-\frac{q_{i}^{-2 m_{i}}}{q_{i}-q_{i}^{-1}} \prod_{p=1}^{m_{i}} \mathbf{v}_{i, p} \cdot \prod_{j=1}^{i-1} \prod_{p=1}^{m_{j}} \mathbf{v}_{j, p}^{a_{j i}} \cdot \\
\sum_{k=1}^{m_{i}} \mathbf{v}_{i, k}^{-2} R_{i}^{(-)}\left(q_{i}^{2} \mathbf{v}_{i, k}^{2}\right) \frac{\prod_{j=1}^{i-1} \prod_{r=1}^{-a_{j i}} \prod_{p=1}^{m_{j}}\left(q_{i}^{2} \mathbf{v}_{i, k}^{2}-q_{j}^{a_{j i}+2 r} \mathbf{v}_{j, p}^{2}\right)}{\prod_{p \neq k}\left(\mathbf{v}_{i, k}^{2}-\mathbf{v}_{i, p}^{2}\right)} \mathbf{u}_{i, k}
\end{gathered}
$$

where $R_{i}^{( \pm)}(z)$ be the polynomials such that $R_{i}^{(+)}(z) R_{i}^{(-)}(z)=\prod_{s=1}^{l_{i}}\left(z \mathbf{w}_{i, s}^{-d}-\mathbf{w}_{i, s}^{d}\right)$ and $c_{\beta_{i}}=$ $\prod_{j=1}^{\ell} q_{j}^{m_{j} n_{j i} / 2}$.

(ii). Let the elements of $\mathbb{T}_{q}$ are represented by (3.7). Then the operators (4.2) define a representation of $U_{q}^{M}(\mathfrak{g})$ in $\mathcal{M}$.

Remark 4.1 In the case $\mathfrak{g}=\mathfrak{s l}(\ell+1)$ the Theorem 4.1 is a natural generalization of the Theorem 3.1 in [16].

Note that there are known several embeddings of $U_{q}(\mathfrak{g})$ into the algebra of quantum torus ( e.g. [2], 24], 25]). Our construction is the new one and has deep relations with 
the Quantum Inverse Scattering Method [14] as well as with the natural parameterization of the moduli spaces of monopoles [17]. The detailed discussion of the connection with the monopoles on $\mathbb{R}^{2} \times S^{1}$ will be described in [18].

\section{References}

[1] M.F. Atiyah, N. Hitchin, The geometry and dynamics of magnetic monopoles, Prinston, NJ University Press (1988).

[2] H. Awata, M. Noumi, S. Odake, Heisenberg realization for $U_{q}\left(s_{n}\right)$ on the flag manifold, Lett. Math. Phys. 30 No.1, (1994), 35-43.

[3] A. Belavin and V. Drinfeld Triangle equations and simple Lie algebras, Funct. Anal. Appl., 16 (1982), 159.

[4] R. Bielawski Asymptotic metrics for $S U(N)$ - monopoles with maximal symmetry breaking. Comm. Math. Phys. 199 (1998), 297-325.

[5] V. Chari, A. Pressley, A guide to quantum groups, Cambridge Univ. Press, Cambridge, 1994.

[6] V.G. Drinfeld, Hopf algebras and the quantum Yang-Baxter equation, Soviet Math. Dokl. 32, 254-258, (1985).

[7] V.G. Drinfeld, A new realization of Yangians and of quantum affine algebras, Dokl. Akad. Nauk SSSR 296 (1987), no. 1, 13-17 (Russian); translated in Soviet Math. Dokl. 36 (1988), 212-216.

[8] L.D. Faddeev, Quantum completely integrable models in field theory, Sov. Sci. Rev., Sect. C (Math. Phys. Rev.) 1 (1980), 107-155.

[9] B. Feigin, A. Odesskii, Vector bundles on Elliptic Curves and Sklyanin Algebras in: Topics in quantum groups and finite type invariants, Mathematics at the Independent University of Moskow, B. Feigin and V. Vasiliev eds, Advances in Mathematical Sciences 38, AMS Translations, ser. 2, 185 (1998), 65-84.

[10] B. Feigin, A. Odesskii, Elliptic deformations of current algebras and their representations by difference operators, Funk. Anal. Appl. 31 (1997), 57-70.

[11] M. Finkelberg, A. Kuznetsov, N. Markarian, I. Mirković, A note on the symplectic structure on the space of G-monopoles, Comm. Math. Phys. 201 (1999), 411-421.

[12] I.M. Gelfand, M.L. Tsetlin, Finite-dimensional representations of the group of unimodular matrices, Dokl.Akad. Nauk SSSR 71 (1950), 825-828 (Russian), translated in I.M. Gelfand, Collected Papers, Vol. II, Springer, Berlin, 1988, 653-656. 
[13] I.M. Gelfand, M.I. Graev, Finite-dimensional irreducible representations of the unitary and the full linear groups, and related special functions, Izv. Akad. Nauk SSSR, Ser.Mat. 29 (1965), 1329-1356; translated in Amer. Math. Soc. Trans. Ser. 264 (1965), $116-146$.

[14] A. Gerasimov, S. Kharchev, D. Lebedev, Representation Theory and Quantum Inverse Scattering Method: The Open Toda Chain and the Hyperbolic Sutherland Model, Int. Math. Res. Notices 17 (2004), 823-854.

[15] A. Gerasimov, S. Kharchev, D. Lebedev, On a class of integrable systems connected with $G L(N, \mathbb{R})$, Int. J. Mod. Phys. A 19 Suppl., (2004), 205-216.

[16] A. Gerasimov, S. Kharchev, D. Lebedev, Representation theory and quantum integrability, arXiv: math.QA/0402112.

[17] A. Gerasimov, S. Kharchev, D. Lebedev, S. Oblezin, On a class of representations of the Yangian and moduli space of monopoles, arXiv: math.AG/0409031.

[18] A. Gerasimov, S. Kharchev, D. Lebedev, S. Oblezin, in preparation.

[19] V. Kac, Infinite dimensional Lie algebras, Third edition, Cambridge University Press, Cambridge, 1990.

[20] S. Kharchev, D. Lebedev, Eigenfunctions of $G L(N, \mathbb{R})$ Toda chain: The Mellin-Barnes representation, JETP Lett. 71 (2000), 235-238.

[21] S. Kharchev, D. Lebedev, Integral representations for the eigenfunctions of quantum open and periodic Toda chains from QISM formalism, J. Phys. A34 (2001), 2247-2258.

[22] P.P. Kulish, E.K. Sklyanin, Quantum spectral transform method. Recent developments, Lecture Notes in Phys. 151, pp. 61-119, Springer, Berlin-New York, 1982.

[23] G. Lusztig, Introduction to quantum groups, Progress in Mathematics, 110, Birkhäuser Boston, Inc., Boston, MA, 1993.

[24] A. Morozov and L. Vinet, Free-Field Representation of Group Element for Simple Quantum Group, Int. J. Mod. Phys. A13 (1998), 1651-1708.

[25] A. Volkov and A. Kashaev From the Tetrahedron Equation to Universal R-Matrices, L. D. Faddeev's Seminar on Mathematical Physics, 79-89, Amer. Math. Soc. Transl. Ser. 2, 201, Amer. Math. Soc., Providence, RI, 2000. 